\title{
Wide Dynamic Range OSNR Monitoring for RZ-DQPSK Systems using Delay-Tap Sampling Technique
}

\author{
F.N. Khan ${ }^{1}$, Alan Pak Tao Lau ${ }^{2}$, Chao Lu ${ }^{1}$ and P.K.A. Wai ${ }^{1}$ \\ ${ }^{1}$ Photonics Research Centre, Dept. of Electronic and Information Engineering, \\ ${ }^{2}$ Photonics Research Centre, Dept. of Electrical Engineering, The Hong Kong Polytechnic University, \\ Hung Hom, Kowloon, Hong Kong. Email: 08901853R@eie.polyu.edu.hk
}

\begin{abstract}
We propose half-symbol delay-tap sampling for OSNR monitoring and extend the monitoring range to 5-45 dB. The proposed method also enables calibration-free monitoring with range of $16-43 \mathrm{~dB}$ at 40 Gbps data rate.

(C)2010 Optical Society of America

OCIS codes: (060.2330) Fiber optics communications; (060.2360) Fiber optics links and subsystems
\end{abstract}

\section{Introduction}

Optical signal-to-noise ratio (OSNR) monitoring is critical for the reliable and stable operation of high speed fiber optic communication networks. The advent of reconfigurable optical add-drop multiplexer (ROADM) further necessitates the need for in-band OSNR monitoring of each individual WDM channel since each channel may traverse different paths and optical amplifiers [1]. Recently, asynchronous delay-tap sampling based techniques have been proposed for OSNR monitoring with the advantages being that they are transparent to data rates and modulation formats and do not require clock recovery [2]. By defining and calibrating a parameter having one-toone relationship with OSNR for each system set-up, Kozicki et al. [3] have experimentally demonstrated an OSNR monitoring technique using delay-tap sampling for $20 \mathrm{Gbps}$ RZ-DQPSK systems, with the monitoring range as low as $6.7 \mathrm{~dB}$. This lower limit is governed by the 'eye-closure' of the delay-tap plot at low OSNR values. One disadvantage of calibration based techniques is that the calibration curve (and hence monitoring range) is dependent on input power level and hence needs to be tracked for accurate OSNR monitoring.

In this paper, we propose the use of half-symbol delay-tap sampling for calibration based in-band OSNR monitoring. We observe that with half-symbol delay-tap plots, the number of occurrences of sample pairs along any radial direction from the origin form a uni-modal distribution as compared to typical bi-modal distributions using other delay values. For low OSNR, such a feature avoids monitoring inaccuracies due to the overlapping of two modes, resulting in a larger dynamic range. We also found that half-symbol delay-tap sampling enables calibrationfree OSNR monitoring through direct estimation of OSNR by measuring the electrical signal and noise powers after $\mathrm{O} / \mathrm{E}$ conversion.

\section{Operating Principle}

The operating principle of delay-tap sampling is shown in Figure 1 (a). A signal with period $T_{\mathrm{s}}$ is asynchronously sampled in pairs with a time delay $\tau$ between the two samples. Plotting the set of sampled pairs produces a delaytap plot such as the one shown in Figure 1 (b). The plot allows the separation of distributions of waveform peak and valley by traversing along the diagonal $D$ of the plot [4]. OSNR is monitored by calculating and calibrating a parameter $F=\left(\mu_{\mathrm{D}_{1}}-\mu_{\mathrm{D}_{0}}\right) /\left(\sigma_{\mathrm{D}_{1}}+\sigma_{\mathrm{D}_{0}}\right)$ having a one-to-one relationship with OSNR, where $\mu_{\mathrm{D}_{1}}, \mu_{\mathrm{D}_{0}}$ are the means while $\sigma_{\mathrm{D}_{1}}, \sigma_{\mathrm{D}_{0}}$ are the standard deviations of the distributions of waveform peak and valley respectively. One disadvantage of this calibration based technique is that the delay-tap plot closes down at low OSNR values resulting in an overlap of distributions of waveform peak and valley, thus limiting the monitoring range. However, for half-symbol delay, an open shape delay-tap plot such as shown in Figure 1 (d) is produced. The sample pairs located along $X$ and $Y$ axes correspond to the waveform peak. We define a parameter $F_{\mathrm{OSNR}}=10 \log \left(\mu_{\mathrm{X}} / \sigma_{\mathrm{X}}\right)$ having one-to-one relationship with OSNR, where $\mu_{\mathrm{X}}$ is the mean while $\sigma_{\mathrm{X}}$ is the standard deviation of the distribution of samples located along $X$ axis as shown in Figure 1(f). By calibrating $F_{\text {OSNR }}$ against OSNR, we can measure OSNR effectively. Since halfsymbol delay-tap plot does not encounter distributions overlap, this calibration based technique can measure $F_{\text {OSNR }}$ even at very low OSNR values.

Half-symbol delay-tap sampling also enables us to perform calibration free OSNR monitoring by making use of the fact that in half-symbol delay-tap plot samples located at various angular positions with respect to the origin correspond to distinct pulse amplitudes. Consider for example the sample pairs $(x, 0)$ located along $X$-axis in 


\section{OMK7.pdf}

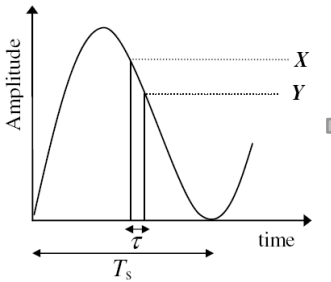

(a)

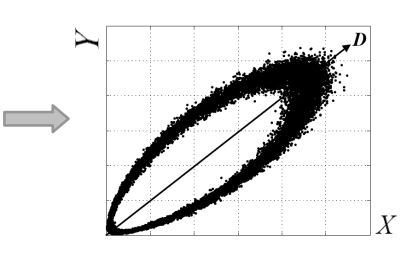

(b)

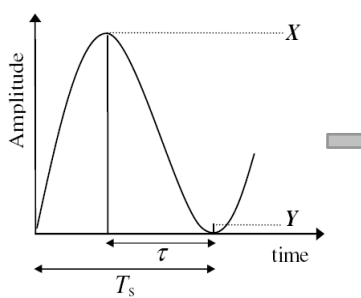

(c)

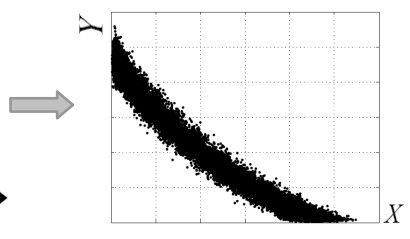

(d)

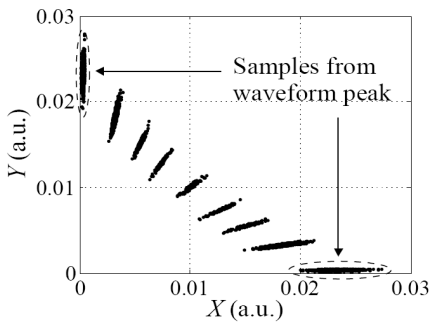

(e)

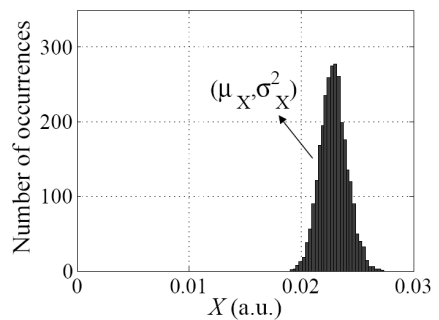

(f)

Fig. 1: (a) Generation of sample pairs with $\tau=T_{\mathrm{s}} / 10$; (b) Typical delay-tap plot for $\tau=T_{\mathrm{s}} / 10$; (c) Generation of sample pairs with $\tau=T_{\mathrm{s}} / 2$; (d) Typical Delay-tap plot for $\tau=T_{\mathrm{s}} / 2$; (e) Extraction of samples at waveform peak; (f) Empirical distribution of samples at waveform peak.

Figure 1 (d) which correspond to the situation when the first sample is located on the waveform peak. We obtain several sets of sample pairs located at distinct angular positions as shown in Figure 1 (e). Assuming Gaussian distributed noise, the averaging of $X$ or $Y$ components of sample pairs in each set will give the signal amplitude at a distinct pulse location. Once several samples of signal pulse are obtained, we can calculate the average electrical signal power $P_{\mathrm{e}}$ from which the optical signal power $P_{\mathrm{Sig}}$ can be obtained using the relation $P_{\mathrm{Sig}}=\sqrt{P_{\mathrm{e}} / R_{\mathrm{L}} R^{2}}$ where, $R$ is the photodiode responsivity and $R_{\mathrm{L}}$ is the load resistance. For the optical noise power measurement we assume that the signal-spontaneous beat noise is much greater than the spontaneous-spontaneous beat noise and receiver noise. The ASE noise power spectral density co-polarized with the signal is given by [5]

$$
N_{\mathrm{ASE}}=\frac{\hat{\sigma}_{\mathrm{x}}^{2}\left[1+B_{\mathrm{s}}^{2} / B_{\mathrm{o}}^{2}+B_{\mathrm{s}}^{2} /\left(4 B_{\mathrm{e}}^{2}\right)\right]^{1 / 2}\left[1+4 B_{\mathrm{e}}^{2} / B_{\mathrm{o}}^{2}\left(1+B_{\mathrm{s}}^{2} / B_{\mathrm{o}}^{2}\right)+2 B_{\mathrm{s}}^{2} / B_{\mathrm{o}}^{2}\right]^{1 / 2}}{4 R^{2} \hat{P}_{\mathrm{Sig}} B_{\mathrm{e}}}
$$

where, $\hat{\sigma}_{\mathrm{X}}^{2}$ is the variance of the signal-spontaneous beat noise at the pulse peak, $B_{\mathrm{s}}, B_{\mathrm{o}}, B_{\mathrm{e}}$ are the bandwidths of the optical signal, optical filter, and electrical filter respectively and $\hat{P}_{\mathrm{Sig}}$ is the peak optical pulse power given by

$$
\hat{P}_{\mathrm{Sig}}=\frac{\mu_{\mathrm{x}}}{R}\left[\left(1+B_{\mathrm{s}}^{2} / B_{\mathrm{o}}^{2}\right)\left(1+B_{\mathrm{s}}^{2} / B_{\mathrm{o}}^{2}+B_{\mathrm{s}}^{2} /\left(2 B_{\mathrm{e}}^{2}\right)\right)\right]^{1 / 2}
$$

where, $\mu_{\mathrm{X}}$ is the average electrical signal at the waveform peak. Both $\hat{\sigma}_{\mathrm{X}}^{2}$ and $\mu_{\mathrm{X}}$ can be calculated from the distribution of samples located along $X$ axis of delay-tap plot and consequently the OSNR can be estimated by $\mathrm{OSNR}=10 \log \left(P_{\mathrm{Sig}} /\left(2 N_{\mathrm{ASE}} B_{\mathrm{o}}\right)\right)$.

\section{Simulation Set-up and Results}

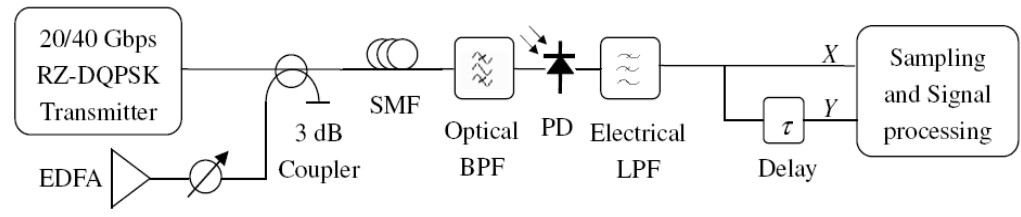

(a)

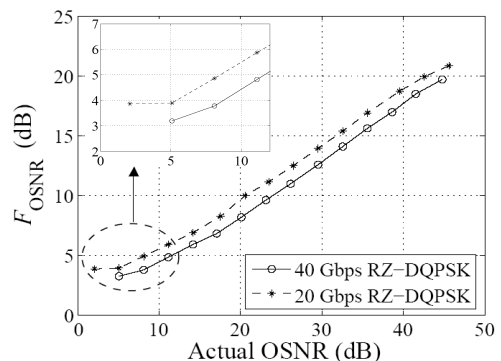

(b)

Fig.2: (a) Simulation set-up for OSNR monitoring using delay-tap sampling; (b) Noise parameter $F_{\text {OSNR }}$ vs. Actual OSNR

The simulation set-up is shown in Figure 2 (a). RZ-DQPSK signals with 50\% duty cycle and data rates of 20 Gbps and $40 \mathrm{Gbps}$ are transmitted over a SMF and an EDFA is used to add variable amount of ASE noise to the signal to adjust the OSNR. At the photodetector output, the electrical signal is splitted into two parts and a half-symbol delay is introduced in one path to generate sample pairs $(X, Y)$. Two channels of an oscilloscope are used to asynchronously sample 17,000 sample pairs which are then processed for OSNR monitoring. The simulation results 


\section{OMK7.pdf}

for calibration based OSNR monitoring using half-symbol delay-tap sampling are shown in Figure 2 (b). It is clear that $F_{\mathrm{OSNR}}$ is sensitive to OSNR values as low as $5 \mathrm{~dB}(0.1 \mathrm{~nm}$ noise bandwidth) for both data rates. In addition, $F_{\text {OSNR }}$ is sensitive to OSNR values up to $45 \mathrm{~dB}$. Hence, half-symbol delay-tap plot offers wider OSNR monitoring range as compared to existing calibration based techniques using smaller delay values.

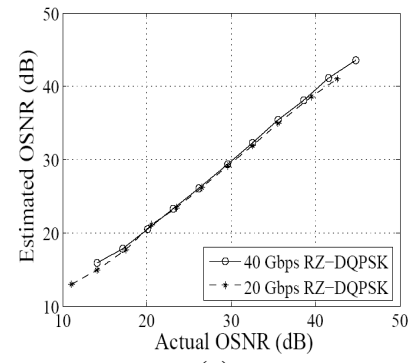

(a)

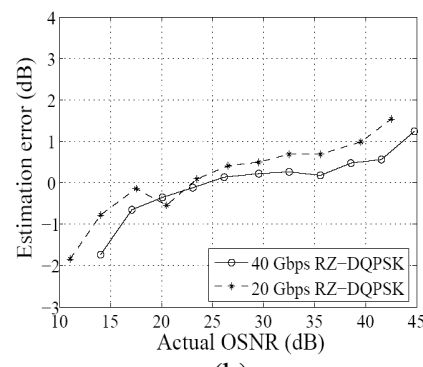

(b)

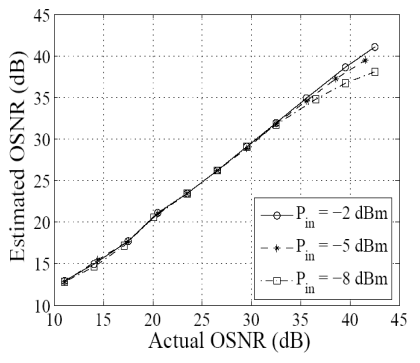

(c)

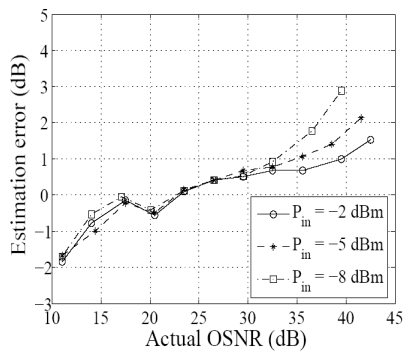

(d)

Fig.3: (a) Actual vs. estimated OSNR for 20 Gbps and 40 Gbps RZ-DQPSK systems; (b) OSNR estimation error; (c) Estimated OSNR for different input power levels for $20 \mathrm{Gbps}$ system; (d) Corresponding estimation error for different input power levels.

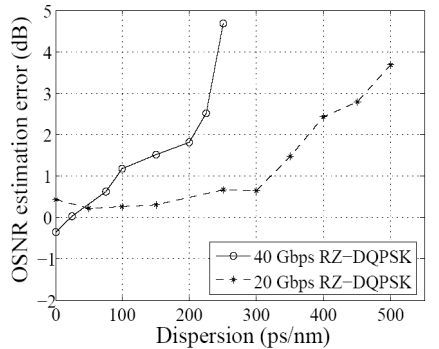

(a)

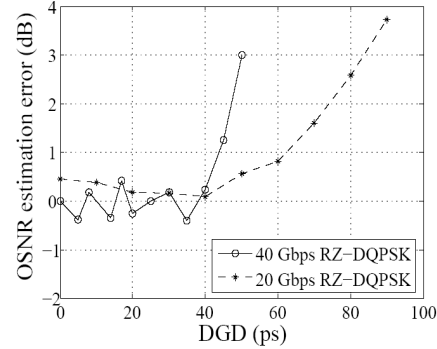

(b)

Fig.4: Effect of (a) CD and (b) PMD on calibration-free OSNR monitoring. The reference OSNR is $23 \mathrm{~dB}$.

Simulation results for calibration-free OSNR monitoring using Equations (1) and (2) are shown in Figures 3 (a) and (b) for an input power of $-2 \mathrm{dBm}$. It is evident that the monitoring range (estimation error $< \pm 1 \mathrm{~dB}$ ) for $20 \mathrm{Gbps}$ is 13 to $40 \mathrm{~dB}$ while that of $40 \mathrm{Gbps}$ is 16 to $43 \mathrm{~dB}$ (in $0.1 \mathrm{~nm}$ bandwidth). The upper OSNR monitoring limit is determined by the receiver thermal and shot noise while the lower monitoring limit is governed by the spontaneousspontaneous beat noise. We also investigated the effect of different input power levels on the monitoring accuracy. As shown in Figures 3 (c) and (d) this technique is relatively insensitive to the input power levels except at high OSNR values. This is due to the fact that when the input power is very low then at high OSNR values the receiver noise dominates the signal-spontaneous beat noise, resulting in estimation errors. Nevertheless, for input power levels as low as $-8 \mathrm{dBm}$ the curves are quite close up till $32 \mathrm{~dB}$ OSNR. This is a big advantage as compared to calibration based techniques where different calibration curves are obtained for different input power levels [3].

Finally, we investigated the effect of CD and PMD on the accuracy of calibration-free OSNR monitoring for a reference OSNR of $23 \mathrm{~dB}$. The results are shown in Figure 4. It is clear that the estimation error increases with an increase in CD or DGD. This is due to the fact that the dispersion causes inter-symbol-interference, resulting in waveform distortion. The OSNR estimation error remains less than $\pm 1 \mathrm{~dB}$ for CD and DGD up to $320 \mathrm{ps} / \mathrm{nm}$ and 62 ps for $20 \mathrm{Gbps}$ while $90 \mathrm{ps} / \mathrm{nm}$ and 43 ps for 40 Gbps respectively. The CD and DGD tolerances may vary depending on the OSNR values.

\section{Conclusion}

In this paper, we proposed the use of half-symbol delay-tap sampling for low cost, wide dynamic range, in-band OSNR monitoring for 20/40 Gbps RZ-DQPSK systems. Half-symbol delay-tap sampling enables both calibration based OSNR monitoring with large dynamic range as well as calibration-free OSNR monitoring which is insensitive to input power levels. Effects of CD and PMD on the OSNR monitoring accuracy are also investigated.

\section{References}

[1] D. C. Kilper et al., IEEE/OSA J. Lightw. Technol., vol. 22, pp.294-304, Jan. 2004.J. Lightwave Tech., vol.22, pp. 294-304, Jan. 2004.

[2] S. D. Dods et al., in Proc. Optical Fiber Commun. Conf. (OFC), 2006, Paper OThP5.

[3] B. Kozicki et al., IEEE Photon. Technol. Lett., vol.21, No.3, pp.179-181, Feb. 2009.

[4] B. Kozicki et al., J. Opt. Netw. 6, pp. 1257-1269, Nov. 2007.

[5] J. Bromage et al., "Raman Amplifiers and Oscillators in Telecommunications," edited by M.N. Islam, Springer Verlag, 2003. 